\title{
Commentary \\ Should all adjuvant breast cancer patients receive a bisphosphonate?
}

\author{
David A Cameron
}

\author{
Senior Lecturer in Medical Oncology, University of Edinburgh \& Western General Hospital, Edinburgh, UK \\ Correspondence: David A Cameron (e-mail: dcameron@srv0.med.ed.ac.uk) \\ Published: 1 October 2003 \\ Breast Cancer Res 2003, 5:296-299 (DOI 10.1186/bcr648) \\ (C) 2003 BioMed Central Ltd (Print ISSN 1465-5411; Online ISSN 1465-542X)
}

\begin{abstract}
This commentary discusses the report by Trevor Powles and colleagues on their double-blind, placebo-controlled randomised trial of 2 years' adjuvant oral clodronate. Although the reported survival benefit in the clodronate-treated group confirms the results of one prior study as well as the generally held view that bisphosphonates could be advantageous, the reviewer does not feel that the data support the universal use of this additional adjuvant therapy.
\end{abstract}

Keywords: adjuvant therapy, bisphosphonate, clodronate, randomised trial

\section{Introduction}

There have been significant improvements in recent years in the outcome for patients with early breast cancer. There is little doubt that the improvements have been brought about by a combination of earlier detection in asymptomatic patients and more aggressive use of anticancer therapies in the absence of clinically evident disease.

In trying to understand why some cancers metastasise to some organs and not others, students are often taught the 'seed and soil' hypothesis of metastatic cancer. This suggests that the ability of cells to survive and grow to produce clinically detectable metastases distant to the site of the primary tumour is a reflection of the properties of both the host tissue/organ and the metastatic tumour cells. However, the majority of successful anticancer therapies to date have in reality only been directed at the seed (the postulated micrometastases that determine the ultimate outcome for our patients) or, in the case of endocrine therapies, the endocrinology of the whole patient. Trevor Powles and colleagues recently presented their study about clodronate in the adjuvant setting [1], reporting an overall survival advantage to be gained by administration of a therapy designed to alter the properties of only one organ in the patient: bone, the 'soil' of any results present us with the question of whether it is time for a change in clinical practice.

\section{Targeting soil and seed}

Powles and colleagues [1] are to be congratulated for taking the paradigm of adjuvant therapy one step further in that, by administering 2 years' therapy with an oral bisphosphonate, they attempted to change the environment in which bone micrometastases persist in order to improve patients' outcome. As such, their study is a milestone that may have profound influence upon future approaches to the treatment of early disease.

Bisphosphonates, among other agents, inhibit osteoclasts $[2,3]$ and thus can potentially disrupt the vicious cycle that probably occurs in the vicinity of bone micrometastases: tumour cells stimulate the osteoclasts, which leads to increased bone resorption and the release of growth factors that in turn stimulate the growth of the metastases. Giving a bisphosphonate in combination with a systemic anticancer therapy, as was the case for $95 \%$ of the patients in the experimental arm, is the epitome of targeting soil and seed at one and the same time. It also exploits the potential synergy with traditional anticancer therapies $[4,5]$ and the possible direct antitumour effects of bisphosphonates [6-8]. The study by Powles and colleagues [1] 
suggests that combination therapy is a better strategy than just targeting the seed with systemic anticancer therapy alone.

\section{Trial summary}

Powles and colleagues' report in the August 2002 issue of the Journal of Clinical Oncology [1] presents the first results from a double-blind randomised trial of 2 years' oral clodronate or placebo in 1069 women with early breast cancer. Patients were expected to have their surgery, radiotherapy, and adjuvant systemic therapy according to local protocols. It was not clear whether any stratification criteria were applied, and the survival analyses were not controlled for the adjuvant systemic therapy given.

The primary end-point was relapse in bone, and there was a non-significant reduction (63 versus 80 patients, $P=0.127$ ). Similarly there were no significant differences in the incidence of non-bone metastases (112 versus 128, $P=0.257)$. There were, however, significantly fewer patients developing bone metastases during the time they took clodronate/placebo (12 versus $28, P=0.016$ ), and there was a significant improvement in overall survival (98 versus 129 deaths, $P=0.047$ ) [1].

\section{Trial results}

Although the primary end-point was not statistically significant, there were two positive secondary end-points whose possible clinical implications must be considered. First, during the 2 years of therapy, the group of patients who took the oral bisphosphonate clodronate in addition to their standard therapy experienced significantly fewer bone metastases. This advantage appeared to be lost during the subsequent follow-up period, however, with as many patients who had taken bisphosphonates developing bone metastases as those who had not.

Second, an overall survival advantage was noted $(P<0.05)$ that began to appear beyond the 2 years of therapy. As Professor Mundy pointed out in the editorial that accompanied Powles and colleagues' article in the Journal of Clinical Oncology [9], not only are the observed effects consistent with data from model systems [10] but, even without hypothesising an effect on nonosseous disease, it is very plausible that the decrease in bone metastases could lead to the overall survival advantage.

\section{Similar studies}

This is not the first study to use adjuvant oral bisphosphonates with the aim to improve the outcome for women with early breast cancer. Two previous smaller studies showed exactly opposite effects from one another using the same drug. In the study reported by Ingo Diel and colleagues [11], 302 women who were found to have breast cancer cells in bone marrow aspirates were randomised to take oral clodronate for 2 years or not. Although a clear benefit has been reported in terms of disease-free survival, the benefit in non-osseous disease reported initially was not seen on longer follow-up [12]. Furthermore, since this study only included women with evidence of micrometastatic breast cancer in the bone marrow, it is not quite the same as a normal adjuvant study where patients are enrolled on the basis of having no evidence of metastases.

In contrast, a similarly modestly sized study by Saarto and colleagues reported no benefit whatsoever in the incidence of bone metastases with 2 years' adjuvant oral clodronate in women with node-positive breast cancer [13]. They even reported a poorer outcome for non-osseous disease that resulted in a worse survival in the women taking clodronate. There are, however, concerns about significant imbalances in the patients in the two arms that could have led to this paradoxical result.

\section{Implications for practice}

Does this mean that all patients with early breast cancer should now receive a bisphosphonate (and perhaps even clodronate in particular) for 2 years over and above their routine therapy? I believe not, despite my enthusiasm for the study by Powles and colleagues [1], and the reasons lie within the study's detail.

A randomised clinical trial of an intervention, such as this, contributes to our understanding but can rarely alone change it, unless the benefit of the intervention is absolutely clear. There is no doubt that the benefit reported in the clodronate-treated group is consistent with the previous positive study by Diel and colleagues [11] (but not that of the apparently less-well balanced Saarto and colleagues' trial [13]), as well as our hypotheses and experimental data regarding how a bisphosphonate could benefit patients at risk of developing bone metastases $[14,15]$. But the null hypothesis of any study is that the observed differences could occur by chance, due to heterogeneity in factors affecting the natural history of the underlying disease. In deciding whether the result of a clinical trial such as this should influence standard therapy, the clinician has to determine whether the differences observed between the two arms are due to the therapeutic intervention, or to random imbalances in the many factors that influence patient outcome in early breast cancer.

Using traditional statistical methodology, the difference in the overall bone metastases-free survival curves in the Powles and colleagues' trial [1] is not statistically significantly different - with $P>0.1$, implying a greater than one in 10 chance of the same difference occurring without any therapeutic intervention. Similarly, the difference in the overall survival curves only just reaches statistical signifi- 
cance at the $5 \%$ level, implying that this could also have arisen without any intervention with a chance of about one in 20. Given that at least three statistical analyses were performed (disease free, bone metastases free and overall survival), then if one had applied the Bonferroni correction as would be conventional for multiple primary end-points, this would reduce the level of significance to less than 0.02 . The overall survival curves would thus no longer be considered statistically significantly different, although there would remain a suggestive trend.

Although the traditional prognostic factors are well balanced, the oestrogen receptor status is unknown in almost one-third of patients. The reader is therefore not entirely sure how well balanced the arms are for this important factor, which relates to outcome, to benefit of adjuvant tamoxifen, and to risk of developing bone metastases. Furthermore, there is also evidence for small differences in the numbers of patients in the two arms who did not receive standard breast cancer therapy, such as the additional seven patients in the control arm who had no local therapy at all. There are also small differences in the numbers of patients receiving various chemotherapy regimens. Whether these differences relate to local practices or to the characteristics of the patients' tumours is of no importance: what does matter is that they indicate the possibility of different prognostic groups within the two arms of the trial.

These differences may seem trivial until one realises that the difference in the bone metastases-free survival curves are created by only 16 fewer patients developing bone metastases during the first 2 years of the trial, with no difference in the numbers beyond then. Furthermore, 17 more patients in the control arm developed visceral metastases, and the shape of the overall survival curves are very similar to those of the non-osseous metastases-free curves (which again do not appear to be statistically significantly different). In complete contrast to the pattern of emergence of the bone metastases, however, the difference in the proportion of patients developing non-osseous metastases only appeared after patients had stopped the clodronate/placebo medication.

These observations suggest two possible hypotheses: (1) either clodronate does have a small effect on the rate of appearance of non-osseous disease, consistent with the first report [11], but not the later report [12], of the Diel and colleagues' study; or (2) there was an imbalance in the as yet undefined factors that determine the appearance of visceral metastases and their poorer outcome.

Since the data presented do not allow us to confirm either of these two hypotheses, the Powles and colleagues' report alone does not provide sufficient grounds for a dronate arm had only two-thirds of the number of nonbreast cancer deaths seen in the control arm: either a further reflection of the heterogeneity in patients even in a study of this size, or perhaps evidence for a survival benefit unrelated to any effect on breast cancer!

\section{Conclusions}

Powles and colleagues clearly present an important study, which may well indicate the direction of future research and practice. If longer follow-up affords an even greater bone metastases-free survival advantage to the clodronate group, a change in practice could be considered. Meanwhile, studies are being planned with more potent bisphosphonates. If the studies show similar or greater benefits, then the case for the use of this class of drugs in routine practice will be made.

\section{Competing interests}

None declared.

\section{Acknowledgement}

The author is grateful to an anonymous reviewer whose comments have, it is hoped, made this article more accessible to non-clinicians.

\section{References}

1. Powles T, Paterson S, Kanis JA, McCloskey E, Ashley S, Tidy A, Rosenqvist K, Smith I, Ottestad L, Legault S, Pajunen M, Nevantaus A, Mannisto E, Suovuori A, Atula S, Nevalainen J, Pylkkanen L: Randomized, placebo-controlled trial of clodronate in patients with primary operable breast cancer [comment]. J Clin Oncol 2002, 20:3219-3224.

2. Green JR, Clezardin P: Mechanisms of bisphosphonate effects on osteoclasts, tumor cell growth, and metastasis [review] [75 refs]. Am J Clin Oncol 2002, 25:S3-S9.

3. Van Beek ER, Lowik CW, Papapoulos SE: Bisphosphonates suppress bone resorption by a direct effect on early osteoclast precursors without affecting the osteoclastogenic capacity of osteogenic cells: the role of protein geranylgeranylation in the action of nitrogen-containing bisphosphonates on osteoclast precursors. Bone 2002, 30:64-70.

4. Jagdev SP, Coleman RE, Shipman CM, Rostami H, Croucher PI: The bisphosphonate, zoledronic acid, induces apoptosis of breast cancer cells: evidence for synergy with paclitaxel. $\mathrm{Br} J$ Cancer 2001, 84:1126-1134.

5. Magnetto S, Boissier S, Delmas PD, Clezardin P: Additive antitumor activities of taxoids in combination with the bisphosphonate ibandronate against invasion and adhesion of human breast carcinoma cells to bone. Int J Cancer 1999, 83:263269.

6. Hiraga T, Williams PJ, Mundy GR, Yoneda T: The bisphosphonate ibandronate promotes apoptosis in MDA-MB-231 human breast cancer cells in bone metastases. Cancer Res 2001, 61: 4418-4424.

7. Senaratne SG, Pirianov G, Mansi JL, Arnett TR, Colston KW: Bisphosphonates induce apoptosis in human breast cancer cell lines. Br J Cancer 2000, 82:1459-1468.

8. Fromigue $\mathrm{O}$, Lagneaux L, Body JJ: Bisphosphonates induce breast cancer cell death in vitro. $J$ Bone Miner Res 2000, 15: 2211-2221.

9. Mundy GR: Bisphosphonates and tumor burden [comment]. $J$ Clin Oncol 2002, 20:3191-3192.

10. Mundy GR, Yoneda T, Hiraga T: Preclinical studies with zoledronic acid and other bisphosphonates: impact on the bone microenvironment [review] [75 refs]. Semin Oncol 2001, 28: 35-44.

11. Diel IJ, Solomayer EF, Costa SD, Gollan C, Goerner R, Wallwiener D, Kaufmann M, Bastert G: Reduction in new metastases in breast cancer with adjuvant clodronate treatment [see comments]. N Engl J Med 1998, 339:357-363. 
12. Diel IJ, Solomayer E, Gollan C, Schutz F, Bastert G: Bisphosphonates in the reduction of metastases in breast cancer results of the extended follow-up of the First study population [abstract]. Proc ASCO 2000, 19:82a.

13. Saarto T, Blomqvist C, Virkkunen P, Elomaa I. Adjuvant clodronate treatment does not reduce the frequency of skeletal metastases in node-positive breast cancer patients: 5-year results of a randomized controlled trial. J Clin Oncol 2001, 19: 10-17.

14. Paterson $\mathrm{AH}$ : The potential role of bisphosphonates as adjuvant therapy in the prevention of bone metastases [review] [62 refs]. Cancer 2000, 88:3038-3046.

15. Yoneda T, Michigami T, Yi B, Williams PJ, Niewolna M, Hiraga T: Actions of bisphosphonate on bone metastasis in animal models of breast carcinoma [review] [41 refs]. Cancer 2000, 88:2979-2988.

\section{Correspondence}

Dr David A Cameron, Senior Lecturer in Medical Oncology, University of Edinburgh \& Western General Hospital, Crewe Road South, Edinburgh EH4 2XU, UK. Tel: +44 131537 2193; fax: +44 131537

1014; e-mail: dcameron@srv0.med.ed.ac.uk 